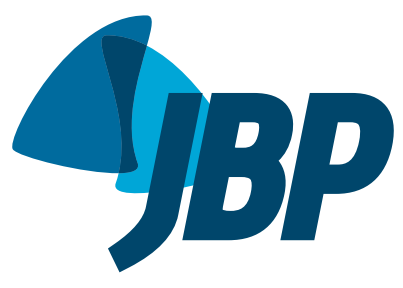

\title{
Hearing thresholds in patients with drug-resistant tuberculosis: baseline audiogram configurations and associations
}

\author{
Olusola Ayodele Sogebi', Muse Olatunbosun Fadeyi², \\ Bolanle Olufunlola Adefuye ${ }^{3}$, Festus Olukayode Soyinka ${ }^{4}$
}

1. ENT Unit, Department of Surgery, College of Health Sciences, Olabisi Onabanjo University, Sagamu, Nigeria.

2. Drug Resistant Tuberculosis Treatment Centre, Sacred Heart Hospital (Special), Lantoro, Abeokuta, Nigeria.

3. Respiratory Unit, Department of Medicine, College of Health Sciences, Olabisi Onabanjo University, Sagamu, Nigeria

4. Ogun State Tuberculosis, Leprosy and Buruli Ulcer Control Program, Ministry of Health, Ogun State, Nigeria.

Submitted: 8 June 2016 Accepted: 31 October 2016.

Study carried out in the Ogun State Drug Resistant Tuberculosis Treatment Center in the Sacred Heart Hospital (Special), Lantoro, Abeokuta, Nigeria.

\begin{abstract}
Objective: To use baseline audiogram parameters in order to ascertain whether drug-resistant tuberculosis (DR-TB) has effects on hearing, as well as to describe the configurations of the audiograms and to determine whether there are parameters that can be associated with those configurations. Methods: This was a prospective study involving patients diagnosed with DR-TB at a tuberculosis treatment center in the state of Ogun, in Nigeria. The patients included in the study were submitted to pure tone audiometry at baseline (within two weeks after treatment initiation). For comparative analyses, data regarding demographic and clinical characteristics were collected from the medical records of the patients. Results: The final sample comprised 132 patients. The mean age of the patients was $34.5 \pm 12.6$ years (range, $8-82$ years), and the male:female ratio was 2:1. Of the 132 patients, 103 (78.0\%) resided in neighboring states, $125(94.7 \%)$ had previously experienced antituberculosis treatment failure, and $18(13.6 \%)$ were retroviral-positive. Normal audiograms were found in 12 patients $(9.1 \%)$, whereas sensorineural hearing loss was identified in 104 (78.8\%), the two most common configurations being ascending, in 54 (40.9\%), and sloping, in $26(19.7 \%)$. Pure-tone averages at low frequencies $(0.25-1.0 \mathrm{kHz})$ and high frequencies $(2.0-8.0 \mathrm{kHz})$ were $33.0 \mathrm{~dB}$ and $40.0 \mathrm{~dB}$, respectively. Regarding the degree of hearing loss in the better ear, 36 patients (27.3\%) were classified as having normal hearing and 67 (50.8\%) were classified as having mild hearing loss (26-40 dB), whereas 29 (21.9\%) showed moderate or severe hearing loss. Among the variables studied (age, gender, retroviral status, previous treatment outcome, and weight at admission), only male gender was associated with audiometric configurations. Conclusions: In this sample of patients with DR-TB, most presented with bilateral, mild, suboptimal sensorineural hearing loss, and ascending/sloping audiometric configurations were associated with male gender.

Keywords: Audiometry, pure-tone; Hearing loss, high-frequency; Drug-related side effects and adverse reactions; Tuberculosis, multidrug-resistant.
\end{abstract}

\section{INTRODUCTION}

Drug-resistant tuberculosis (DR-TB) refers to infection with an isolate of Mycobacterium tuberculosis that is resistant to one of the first-line antituberculosis drugs, namely isoniazid, rifampin, pyrazinamide, ethambutol, and streptomycin. On the basis of their degree of resistance to such medications, mycobacteria can be classified as single drug-resistant, multidrug-resistant (MDR), or extensively drug-resistant. The reported prevalence of DR-TB is variable, strains resistant to isoniazid plus rifampin (i.e., MDR-TB strains) being identified in $0.6 \%$ to $2.9 \%$ of newly diagnosed cases of tuberculosis in sub-Saharan Africa. ${ }^{(1,2)}$ In addition, DR-TB is common among retroviral-infected individuals; the incidence of rifampin-resistant tuberculosis among HIV-infected individuals in Nigeria was $7.0 \%$ over a period of three years (2009-2012). ${ }^{(3)}$ The emergence of DR mycobacterial strains is an important factor that fuels the tuberculosis epidemic and its associated morbidity and mortality. ${ }^{(4)}$
Although DR-TB can be a primary infection when a patient acquires a $M$. tuberculosis strain that is already resistant, it arises more often through the selection of mutated strains by inadequate therapy. The most powerful predictor of the presence of DR-TB is a history of a previous antituberculosis treatment. (5) Other factors, such as a shortage of drugs, leads to inadequacy of initial antituberculosis regimen and fuels the emergence of DR-TB, especially in resource-poor settings. Furthermore, increased costs of treatment and complications associated with the usage of antituberculosis medications considerably contribute to the complexity of DR-TB treatment. ${ }^{(5)}$

The adverse effects of antituberculosis treatment include skin rashes, gastrointestinal disturbances, and hepatotoxicity, whereas aminoglycosides can cause nephrotoxicity and ototoxicity. ${ }^{(6)}$ Sensorineural hearing impairment has been noted as the most common form of manifestation of ototoxicity. ${ }^{(7)}$ This is detected by monitoring hearing threshold parameters in patients on antituberculosis drugs as part of the DR-TB management 
protocol. Hearing impairment resulting from ototoxicity is detected by comparing hearing threshold measurements during treatment with an initial hearing assessment obtained from the patient on admission prior to the commencement of therapy (baseline audiometry). However, tuberculosis can affect the ears in other ways as part of its extrapulmonary manifestations, causing infections, such as tuberculous otitis media, facial nerve paralysis, and various types and degrees of hearing loss $(\mathrm{HL}){ }^{(8)}$ The effects that mycobacteria have on hearing, unlike the effects of the medications, can be assessed subjectively from baseline audiometry obtained prior to treatment initiation.

The objective of the present study was to use baseline audiogram parameters in order to ascertain whether DR-TB has effects on hearing, to describe the configurations of the audiograms, and to determine whether there are clinical parameters that can be associated with those configurations. We believe this will assist in the initial characterization of the hearing thresholds and clarify the audiological effects of tuberculosis.

\section{METHODS}

This was a prospective study involving patients followed at the Ogun State Drug Resistant Tuberculosis Center in the Sacred Heart Hospital (Special), located in Lantoro, Abeokuta, Nigeria, between November of 2014 and October of 2015. The center receives DR-TB patients from its home state of Ogun and from neighboring states (Lagos, Oyo, Ondo, Ekiti, Kwara, Kogi, Edo, and Delta).

The patients who were diagnosed with DR-TB-by means of GeneXpert MTB/RIF ${ }^{\circledR}$ (Cepheid, Sunnyvale, CA, USA) and a positive sputum culture for M. tuberculosis-were admitted and treated at the center. The cases were managed by a team of experts, which included a pulmonologist, an otolaryngologist, clinicians (trained in tuberculosis management), a psychiatrist, nurses, and a social worker. The pulmonologist performed the initial clinical assessments of the patients and confirmed the diagnosis of DR-TB aided by the investigations. Other experts also performed different roles in the management of the cases. The study protocol was approved by the Research Ethics Committee of the Sacred Heart Hospital, and all participating patients gave written informed consent.

As part of the management protocol for DR-TB, the otolaryngologist evaluated the initial (baseline) audiometry of the patients at or within two weeks after their admission and treatment initiation in order to assess their hearing thresholds. The rationale for and significance of such assessments were explained to the patients, and only those who consented had their data included in the study. Patients were questioned concerning ear diseases-present, previous or recurrent ear discharges-symptoms- $\mathrm{HL}$, noise in the ears (tinnitus), echoes, and history of vertigo-and self-estimation of hearing-good or poor-in order to detect hearing impairments and difficulty in hearing.
The two ears were thereafter examined using an otoscope (HEINE Optotechnik, Herrsching, Germany) in order to detect pathologies, such as perforated tympanic membrane, ear discharge, wax impaction, and presence of a foreign body. The patients with ear lesions, such as wax impaction, were treated before their hearing assessment was carried out.

The inclusion criteria were having anatomically normal ears or presenting with structural abnormalities resulting from the tuberculosis infection, as well as having performed baseline audiometry within two weeks after treatment initiation. Data for patients with a sequel of perforated tympanic membrane suspected to be related to suppurative otitis media and not resulting from the tuberculosis infection were excluded from the study.

Each patient was seated in a quiet room with an ambient noise level of $27 \mathrm{~dB}$ (sound pressure level), was instructed on how to perform the test, and was told what type of responses would be expected. A calibrated diagnostic audiometer (Amplivox 240; Amplivox Ltd., Eynsham, UK), a head band, and a bone stimulator were used in order to produce pure-tone sounds, ranging from $0.25-8.00 \mathrm{kHz}$ and $0.25-4.0 \mathrm{kHz}$, for the determination of air conduction and bone conduction thresholds, respectively. Hearing thresholds were measured as sound intensity in decibels based on consistent responses of the patient at each frequency tested. The responses were plotted as a chart in order to produce the audiogram for each ear. The types and laterality of the audiograms were also recorded. The types of audiogram were classified as normal, conductive $\mathrm{HL}$, sensorineural $\mathrm{HL}$, or mixed HL. Sensorineural HL was subclassified as sloping, flat, or ascending, based on the form of the tracings moving from the lower to the higher frequencies on the audiograms. Laterality was based on the side of the abnormal audiogram, and a discordant audiogram represented different types of abnormalities in the two ears. The degree of $\mathrm{HL}$ was also noted in the better ear in each patient. We adopted the World Health Organization classification of $\mathrm{HL}_{,}{ }^{(9)}$ using the pure-tone average, as follows: 0-25 dB, normal hearing; 26-40 $\mathrm{dB}$, mild $\mathrm{HL}$; 41-60 dB, moderate $\mathrm{HL}$; $61-80 \mathrm{~dB}$, severe $\mathrm{HL}$; and $\geq 81 \mathrm{~dB}$, profound $\mathrm{HL}$.

Demographic parameters, such as usual place of domicile, and clinical parameters, including a history of tuberculosis treatment (cure or failure) and retroviral status, were retrieved from the medical records of the patients. These and the audiometric data were entered into a spreadsheet and analyzed using the IBM SPSS Statistics software package, version 20.0 (IBM Corporation, Armonk, NY, USA). The descriptive characteristics of the patients were summarized in tables and presented as absolute numbers and proportions (categorical variables) or as means and standard deviations (continuous variables). Variables (age, gender, retroviral status, previous treatment failure, and body weight) were compared with the audiogram configurations of the patients. The chi-square test was 
used in order to detect significant differences between the variables $(p<0.05)$.

\section{RESULTS}

Over a period of one year, 142 patients were followed. However, 10 patients were excluded from the study ( 5 were unable to perform baseline audiometry on account of being too weak to withstand the stress of audiometric assessment; 2 presented with inconsistent, unreliable responses during audiometry; and 3 underwent audiometry after two weeks of treatment). Therefore, the sample comprised 132 patients. The mean age of the patients was $34.5 \pm 12.6$ years (range, $8-82$ years). The male:female ratio was $2: 1$. Of the 132 patients, $103(78.0 \%)$ did not reside in Ogun state and 18 $(13.6 \%)$ were retroviral-positive. Self-reported hearing status was as follows: 111 patients (84.1\%) described their hearing as good; 13 (9.8\%) were undecided, and $8(6.1 \%)$ described it as poor. Failure of previous tuberculosis treatment (with different combinations of drugs, including rifampin, isoniazid, pyrazinamide, and ethambutol) was reported by 125 patients (94.7\%). After two months of first-line treatment, there was no sputum conversion in 44 patients $(33.3 \%)$, whereas, after three months of first-line treatment, there was neither sputum conversion nor clinical improvement despite good treatment compliance in 75 patients $(56.8 \%)$, as shown in Table 1.

Table 2 presents the audiometric characteristics of the patients, showing that $<10 \%$ of the patients had normal audiograms. Sensorineural HL was identified in 104 patients $(78.8 \%)$, among whom the most common configurations were ascending (seen in 40.9\%) and sloping (seen in $19.7 \%$ ). The pure-tone averages (arithmetic mean of air conduction thresholds) at low frequencies $(0.25-1.0 \mathrm{kHz})$ and high frequencies (2.0$8.0 \mathrm{kHz}$ ) were $33.0 \mathrm{~dB}$ and $40.0 \mathrm{~dB}$, respectively. The analysis of the degree of $\mathrm{HL}$ in the patients revealed that $27.3 \%$ had normal hearing in the better ear, half (50.8\%) of the patients had mild $\mathrm{HL}(26-40 \mathrm{~dB})$, and the remaining patients had higher degrees of $\mathrm{HL}$.

In Table 3, the factors that could be associated with ascending and sloping audiogram configurations were explored. Among all of the factors studied, only male gender was associated with both audiometric configurations, whereas age, positive retroviral status, previous treatment failure, and body weight $<50.0 \mathrm{~kg}$ at admission were not associated with the audiometric configurations.

\section{DISCUSSION}

The present study has revealed that only 12 $(9.1 \%)$ of our DR-TB patients had normal hearing in both ears, $36(27.3 \%)$ had normal hearing in one ear, and the majority of the patients had hearing impairment in both ears. The main type of hearing impairment was sensorineural $\mathrm{HL}$-especially showing ascending or sloping audiogram configurations, of mild magnitude-which was associated with the male gender. Thus, the prevalences of increased hearing thresholds, i.e. reduced hearing at the baseline level in one ear and in both ears were $90.9 \%$ and $72.7 \%$, respectively. These prevalences are certainly very high, arousing suspicion of a direct effect of mycobacteria on the ears of DR-TB patients. However, this should be interpreted with some caution, since such results might be a reflection of the general trend of hearing in that community, especially because there were no

Table 1. Clinical and demographic characteristics of the patients.

\begin{tabular}{|c|c|c|}
\hline Characteristic & $\mathbf{n}$ & $\%$ \\
\hline Age, years ${ }^{a}$ & $8-82$ & $34.5 \pm 12.6$ \\
\hline \multicolumn{3}{|l|}{ Gender } \\
\hline Male & 87 & 65.9 \\
\hline Female & 45 & 34.1 \\
\hline \multicolumn{3}{|l|}{ Place of residence } \\
\hline Ogun state (rural) & 12 & 9.1 \\
\hline Ogun state (urban) & 17 & 12.9 \\
\hline Outside Ogun state & 103 & 78.0 \\
\hline Weight at admission, $\mathrm{kg}^{\mathrm{a}}$ & $17.0-85.0$ & $50.6 \pm 10.1$ \\
\hline \multicolumn{3}{|l|}{ Retroviral status } \\
\hline Negative & 114 & 86.4 \\
\hline Positive & 18 & 13.6 \\
\hline \multicolumn{3}{|c|}{ Previous antituberculosis treatment failure } \\
\hline Treatment naive & 7 & 5.3 \\
\hline CAT I & 44 & 33.3 \\
\hline CAT II & 75 & 56.8 \\
\hline Others & 6 & 4.6 \\
\hline
\end{tabular}

CAT I: category I represents no sputum conversion for two consecutive months after the end of the second month of first-line treatment or no clinical improvement despite good drug compliance; and CAT II: category II represents no sputum conversion for consecutive months after the end of the third month of first-line treatment or no clinical

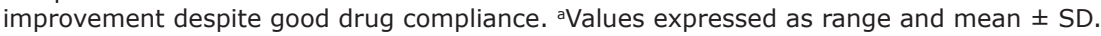


Table 2. Audiometric profile of the patients.

\begin{tabular}{|ccc}
\hline \multicolumn{1}{c}{ Profile } & $\mathbf{n}$ & $\%$ \\
\hline Audiogram laterality & 8 & 6.1 \\
Right-sided & 5 & 3.8 \\
Left-sided & 115 & 87.1 \\
Bilateral & 4 & 3.0 \\
Discordant & & 9.1 \\
Type & 12 & 4.5 \\
Normal & 6 & 78.8 \\
Conductive hearing loss & 104 & 18.2 \\
Sensorineural hearing loss & 24 & 40.9 \\
$\quad$ Flat audiogram configuration & 54 & 19.7 \\
$\quad$ Ascending audiogram configuration & 26 & 7.6 \\
$\quad$ Sloping audiogram configuration & 10 & \\
Mixed hearing loss & & 27.3 \\
Degree of hearing loss in the better ear & 36 & 50.8 \\
Normal hearing & 67 & 18.9 \\
Mild & 25 & 3.0 \\
Moderate & 4 & \\
Severe & & \\
\hline
\end{tabular}

Table 3. Factors associated with ascending and sloping audiogram configurations.

\begin{tabular}{lcccc}
\multicolumn{1}{c}{ Factor } & Ascending audiogram configuration, & $\chi^{2}$ & p \\
& No & Yes & & 0.391 \\
Age $\leq 33$ years & 44.7 & 9.1 & 0.752 & 0.036 \\
Male gender & 49.2 & 16.7 & 5.042 & 0.805 \\
HIV positive & 10.6 & 3.0 & 0.322 & 0.919 \\
Previous treatment failure & 48.5 & 12.1 & 0.498 & 0.624 \\
Weight < 50.0 kg & 41.7 & 8.3 & 0.945 & $\mathbf{p}$ \\
\multicolumn{1}{r}{ Factor } & Sloping audiogram configuration, \% & $\chi^{2}$ & 0.160 \\
Age $\leq 33$ years & No & Yes & 1.972 & 0.037 \\
Male gender & 30.3 & 15.9 & 4.360 & 0.121 \\
HIV positive & 43.2 & 22.7 & 3.814 & 0.814 \\
Previous treatment failure & 10.6 & 3.0 & 2.075 & 0.704 \\
Weight < 50.0 kg & 37.1 & 23.5 & 0.703 & \\
\hline
\end{tabular}

controls for comparison. Moreover, noise pollution levels in that community are high, ${ }^{(10,11)}$ and this might have a bearing on the average hearing thresholds in that population.

A vast majority (94.7\%) of our patients had previous drug treatment failures. The treatment regimens previously used, which varied in content and duration of use, included the standard first-line antituberculosis drugs used in Nigeria (rifampin, isoniazid, pyrazinamide, and ethambutol). These medications were used in a six-month regimen, comprising a two-month intensive phase and a four-month continuation phase. The possibility that previous hearing problems could be worsened by the use of aminoglycosides is an issue to be explored. Researchers had reported an incidence of ototoxicity of up $1.7 \%$ among tuberculosis patients on standard antituberculosis drug combination treatment. ${ }^{(12)}$ Therefore, the increased hearing thresholds found in the present study might be partly related to ototoxic effects of a previous treatment with rifampin or isoniazid.
The reported prevalence of ototoxicity among DR-TB patients under treatment in Europe and Asia was $28.0 \%{ }^{(13)}$ and $70.1 \%,{ }^{(14)}$ respectively. It is noteworthy that those quoted figures are not baseline audiometric values, and ototoxicity is based on stringent criteria, including audiometric permanent hearing threshold shifts, at particular frequencies. It may suffice to posit that the level of hearing of most of the DR-TB patients in our cohort was suboptimal. However, a more serious concern regarding such high prevalences of suboptimal hearing is the long-term effects and repercussions they could have on the hearing of patients who are still going to be on aminoglycoside therapy for months. There is a tendency for $\mathrm{HL}$ to become aggravated, with consequent increases in the proportion of patients with hearing impairment. Considering other medications aside from aminoglycosides in the treatment of tuberculosis is expedient, and research is ongoing in this respect.

Most audiologists define the "baseline" audiogram as one obtained within two weeks after the initiation 
of treatment. ${ }^{(15)}$ This is based on the assumption that significant threshold shifts would not have occurred within two weeks, even if patients had started therapy. In addition, it may not be possible to perform this test immediately after admission due to logistics, and it is better not to delay the initiation of therapy in our local setting. Despite the exclusion of the data of the patients with obvious middle ear disease, such as recurrent ear discharge and perforated tympanic membranes due to suppurative ear infections, $4.5 \%$ of the patients presented with audiometric evidence of conductive $\mathrm{HL}$. This might be due to subclinical malfunctioning of the middle ear conducting mechanisms. The most common type of audiometric pattern found in our sample was sensorineural $\mathrm{HL}$, which denoted the affectation of the hair cells or the cochlear nerves. Tuberculosis has been reported to affect peripheral nerves, causing neuropathies, especially during the late stages of infection, although the pathogenesis of this aspect of the disease remains controversial. ${ }^{(16)}$ However, various reports concerning differential affectation of cochlear hair cells are related to pathologies due to drug-induced ototoxicity, as well as to age-related and noise-induced $\mathrm{HL}$.

In considering audiograms, three configurations of sensorineural $\mathrm{HL}$ have been described, namely ascending, flat, and sloping configurations. In our study, the most common configuration was the ascending configuration, with low-frequency HL (LFHL), which is also called reverse slope audiogram. The LFHL denotes affectation of hair cells at the apical turn of the cochlea. This type of $\mathrm{HL}$ is not easily identified symptomatically in a clinic setting, because the symptoms are subtle, and audiologists therefore often describe it as asymptomatic. Low-frequency sounds are more intense and suspiciously carry less information than do high-frequency sounds. ${ }^{(17)}$ A person with a moderate degree of LFHL may not exhibit any outward sign of $\mathrm{HL}$, such as missing speech sounds or aberrant speech production patterns. Such patients thus maintain a relatively intact intelligibility. Furthermore, low-frequency information may be carried by high-frequency fibers through temporal coding, limiting suggestive clues to LFHL to subtle symptoms, such as difficulties in hearing in a group setting or in a noisy place. Notable causes and associated factors for LFHL include a mutation of the Wolfram syndrome gene 1 (an autosomal recessive disorder called Wolfram syndrome 1$){ }^{(18)}$ Mondini dysplasia, ${ }^{(19)}$ sudden $\mathrm{HL}_{\boldsymbol{I}}{ }^{(20)}$ Ménière's disease, and viral infections. ${ }^{(21)}$. It might be precipitous to assume that mycobacteria affect the hair cells in a manner similar to that of viral infections, and this phenomenon therefore needs further clarification.

The sloping audiogram configuration, which represents high-frequency $\mathrm{HL}$ (HFHL), has been associated with noise-induced hearing impairment and age-related $\mathrm{HL}$ (presbycusis). Although some of the patients in our sample were elderly ( $\geq 60$ years of age), not all of the patients in this age group had HFHL. However, age-related hearing changes tend to start manifesting about the fifth decade of life in Nigerians. (22) Advancing age and the high level of noise in our community might have caused HFHL in some of the patients in our sample. The bulk of the patients had the same pattern in both ears, suggesting similar pathologies. Although tuberculosis primarily affects the lung parenchyma, its effects on other organs of the body are usually systemic. The systemic effects could be due to immune-mediated or vasculitis-related cytopathy, ${ }^{(16)}$ rather than to the direct invasion of mycobacteria in the ears.

Most audiologists are conversant with the World Health Organization classification of the degree of $\mathrm{HL} .{ }^{(9)}$ In the present study, most of the patients had a mild degree of $\mathrm{HL}$, which might not be noticeable at the outset. Continuous exposure to the same stimuli leads to progression and worsening of the clinical condition of patients, whereas the administration of injectable aminoglycosides will lead to the aggravation of the symptoms. Commencing as an initial temporary threshold shift, ototoxicity ultimately leads to a permanent threshold shift, which is irreversible. It is thus suggested that patients with any form of $\mathrm{HL}$ should not take the highly ototoxic aminoglycosides from the outset, or, alternatively, such patients should have more frequent audiometric monitoring in order to detect threshold shifts rapidly and to make adjustments accordingly before a permanent threshold shift sets in. However, the practicability of these suggestions in resource-poor environments is doubtful. Nevertheless, the present study underscores the necessity of initial audiometric hearing threshold measurements. We advocate serial audiometric measurements for all patients under therapy for any form of tuberculosis infection.

While medical litigation against the healthcare giver may not be particularly common in our environment for now, the possibility cannot be dismissed. There were some patients with $\mathrm{HL}$ that will necessitate sound amplification, assistive hearing devices, or aural rehabilitation. Such patients should be started on therapy with a drug such as capreomycin, which appears to have less toxicity ${ }^{(13)}$ than other aminoglycosides, such as kanamycin. However, such patients need to be educated about their hearing problems, the pre-existence of the $\mathrm{HL}$ before the initiation of therapy should be emphasized, and necessary actions will need to be taken. The readily available assistance we rendered to such patients included hearing aids for free.

Discovering factors associated with a disease can aid the clinician in the disease management. Among the factors that were explored concerning the two most common audiometric configurations in the present study, only male gender was found to be associated with both ascending and sloping audiometric configurations. Although this may portend some hormonal influence, that might be oversimplifying the situation. Some studies noted comparatively increased hearing thresholds in males (in comparison with females), especially in those between 20 and 69 years of age.(23-25) Park et al. reported that hearing thresholds at frequencies of 3 
$\mathrm{kHz}, 4 \mathrm{kHz}$, and $6 \mathrm{kHz}$ showed a statistical difference between the two genders for people over 30 years of age, the $4-\mathrm{kHz}$ frequency accounting for the largest significant difference in a population sample in South Korea. (26) The factors that have been attributed to this include occupational and leisure exposure to noise, as well as the use of analgesics or nonsteroidal anti-inflammatory drugs, which more commonly occur in males. ${ }^{(27)}$

None of the other factors studied was found to be associated with either ascending or sloping configurations. Theoretically, it should be expected that older patients, those with previous drug regimen failure, those with lower body weight at admission, and those coinfected with HIV will present with worse hearing thresholds. It was reported, however, that the absolute risks and risk factors for adverse events (possibly including $\mathrm{HL}$ ) were similar between HIV-infected and HIV-uninfected patients treated for DR-TB in a cohort of 57 patients in Namibia. ${ }^{(28)}$ It has been reported that hearing deficits in HIV-infected individuals could be a central (rather than a peripheral) auditory change that pure tone audiogram detects. ${ }^{(29)}$ Similarly, antiretroviral-naïve HIV-infected patients are not more likely to show an increase in having severe adverse drug reactions when on a second-line antituberculosis regimen. ${ }^{(30)}$ This underscores the need for further research into the subject, especially regarding the link resulting from DR-TB and HIV infection.

The factors associated with hearing impairment and audiometric configurations in DR-TB patients can be clarified further by means of randomized controlled studies. Such studies should carry out comparative analyses among normal individuals, patients with tuberculosis, and those with DR-TB. The lack of this type of analysis remains the major limitation of the present study. It is also noteworthy that evaluating the body mass index of the patients at admission would have represented the nutritional status better than their body weight. ${ }^{(31)}$ In addition, the baseline audiometric assessment should be ideally performed before the commencement of therapy. These limitations notwithstanding, the present study suggests that, at presentation, most of the patients with DR-TB in Nigeria had mild, suboptimal, sensorineural hearing levels bilaterally and ascending or sloping audiometric configurations, the male gender being the only factor associated with such configurations.

\section{REFERENCES}

1. Sanchez-Padilla E, Ardizzoni E, Sauvageot D, Ahoua L, Martin A Varaine $F$, et al. Multidrug- and isoniazid-resistant tuberculosis in three high HIV burden African regions. Int J Tuberc Lung Dis. 2013;17(8):1036-42. https://doi.org/10.5588/ijtld.12.0842

2. Nigeria. Federal Ministry of Health. National DR-TB prevalence survey report. Abuja: Nigeria; 2011. p. 14-5.

3. Dinic L, Akande P, Idigbe EO, Ani A, Onwujekwe D, Agbaji O, et al. Genetic determinants of drug-resistant tuberculosis among HIV infected patients in Nigeria. J Clin Microbiol. 2012;50(9):2905-9. https://doi.org/10.1128/JCM.00982-12

4. Singh N, Sidiq Z, Bhalla M, Myneedu VP, Sarin R. Multi-drug resistant tuberculosis among category | treatment failures-a retrospective study. Indian J Tuberc. 2014;61(2):148-51

5. Marahatta SB Multi-drug resistant tuberculosis burden and risk factors: an update. Kathmandu Univ Med J (KUMJ). 2010;8(29):11625. https://doi.org/10.3126/kumj.v8i1.3234

6. Zhang $H Q$, Xi XE, Wang $Y L$, Han W, Zhang CX, Jiao JH. Side effects of tuberculosis treatment with fixed-dose combinations. J Biol Regul Homeost Agents. 2015;29(2):379-88.

7. Vasconcelos KA, Lima MA, Frota S, Ruffino Netto A, Kritski AL. Audiometric evaluation of patients treated for pulmonary tuberculosis. J Bras Pneumol. 2012;38(1):81-7. https://doi.org/10.1590/S180637132012000100012

8. Akkara SA, Singhania A, Akkara AG, Shah A, Adalja M, Chauhan N A Study of Manifestations of Extrapulmonary Tuberculosis in the ENT Region. Indian J Otolaryngol Head Neck Surg. 2014;66(1):46-50. https://doi.org/10.1007/s12070-013-0661-7

9. World Health Organization [homepage on the Internet]. Geneva: World Health Organization [cited 2016 Jun 1]. Prevention of blindness and deafness. Grades of hearing impairment [About 2 screens]. Available from: http://www.who.int/pbd/deafness/hearing_ impairment_grades/en/

10. Sogebi OA, Amoran OE, Iyaniwura CA, Oyewole EA. Awareness and attitudes to noise and its hazards in motor parks in a sub-urban Nigerian town. Niger Postgrad Med J. 2014;21(1):40-5

11. Oyedepo OS, Saadu AA. A comparative study of noise pollution levels in some selected areas in Ilorin Metropolis, Nigeria. Environ Monit Assess. 2009;158(1-4):155-67. https://doi.org/10.1007/ s10661-008-0570-5

12. Gülbay BE, Gürkan OU, Yildiz OA, Onen ZP, Erkekol FO, Baççioğlu $A$, et al. Side effects due to primary antituberculosis drugs during the initial phase of therapy in 1149 hospitalized patients for tuberculosis.
Respir Med. 2006;100(10):1834-42. https://doi.org/10.1016/j. rmed.2006.01.014

13. Sturdy A, Goodman A, José RJ, Loyse A, O'Donoghue M, Kon OM et al. Multidrug-resistant tuberculosis (MDR-TB) treatment in the UK: a study of injectable use and toxicity in practice. J Antimicrob Chemother. 2011;66(8):1815-20. https://doi.org/10.1093/jac/dkr221

14. Javadi MR, Abtahi B, Gholami K, Safari Moghadam B, Tabarsi P Salamzadeh J. The Incidence of Amikacin Ototoxicity in MultidrugResistant Tuberculosis Patients. Iran J Pharm Res. 2011;10(4):90511.

15. Duggal $P$, Sarkar M. Audiologic monitoring of multi-drug resistant tuberculosis patients on aminoglycoside treatment with long term follow-up. BMC Ear, Nose Throat Disord. 2007; 7:5. https://doi. org/10.1186/1472-6815-7-5

16. Warpe BM, Poflee SV, Pande NP, Shrikhande AV. Tuberculous neuritis: a rare sequel of a common disease. Indian J Pathol Microbiol. 2014;57(1):69-71. https://doi.org/10.4103/0377-4929.130902

17. Salminen NH. Human cortical sensitivity to interaural leve differences in low- and high-frequency sounds. J Acoust Soc Am. 2015;137(2):EL190-3. https://doi.org/10.1121/1.4907736

18. Gene identified for low-frequency hearing loss. Hear J. 2002;55(3):7 8. https://doi.org/10.1097/01.HJ.0000293475.07402.ee

19. Parving A. Inherited low-frequency hearing loss. A new mixed conductive/sensorineural entity? Scand Audiol. 1984;13(1):47-56. https://doi.org/10.3109/01050398409076257

20. Mattox D, Simmons E. Natural history of sudden sensorineura hearing loss. Ann Otol Rhinol Laryngol. 1977;86(4 Pt 1):463-80. https://doi.org/10.1177/000348947708600406

21. Djupesland G, Flottorp G, Degré M, Stien R, Skrede S. Cochlear hearing loss and viral infection. Acta Otolaryngol. 1979;87(3-4):24754. https://doi.org/10.3109/00016487909126416

22. Sogebi OA, Olusoga-Peters OO, Oluwapelumi O. Clinical and audiometric features of presbycusis in Nigerians. Afr Health Sci. 2013;13(4):886-92. https://doi.org/10.4314/ahs.v13i4.4

23. Dreisbach LE, Kramer SJ, Cobos S, Cowart K. Racial and gender effects on pure-tone thresholds and distortion-product otoacoustic emissions (DPOAEs) in normal hearing young adults. Int J Audiol. 2007;46(8):419-26. https://doi.org/10.1080/14992020701355074

24. Bahng J, Lee J. Hearing Thresholds for a Geriatric Population Composed of Korean Males and Females. J Audiol Otol. 2015;19(2):91-6. https://doi.org/10.7874/jao.2015.19.2.91

25. Baraldi Gdos S, de Almeida LC, Borges AC. Hearing loss in aging. 
Braz J Otorhinolaryngol. 2007;73(1) :58-64. https://doi.org/10.1016/ S1808-8694(15)31123-X

26. Park YH, Shin SH, Byun SW, Kim JY. Age- and Gender-Related Mean Hearing Threshold in a Highly-Screened Population: The Korean National Health and Nutrition Examination Survey 2010-2012. PLoS One. 2016;11(3):e0150783. https://doi.org/10.1371/journal. pone. 0150783

27. Dalton DS, Cruickshanks KJ, Wiley TL, Klein BE, Klein R, Tweed TS Association of leisure-timenoise exposure and hearing loss. Audiology. 2001; 40(1):1-9. https://doi.org/10.3109/00206090109073095

28. Sagwa E, Ruswa N, Musasa JP, Mantel-Teeuwisse AK. Adverse events during treatment of drug-resistant tuberculosis: a comparison between patients with or without human immunodeficiency virus co-infection. Drug Saf. 2013;36(11):1087-96. https://doi.org/10.1007/ s40264-013-0091-1

29. Maro II, Moshi N, Clavier OH, MacKenzie TA, Kline-Schoder RJ, Wilbur JC, et al. Auditory impairments in HIV-infected individuals in Tanzania. Ear Hear. 2014;35(3):306-17. https://doi.org/10.1097/01. aud.0000439101.07257.ed

30. Van der Walt M, Lancaster J, Odendaal R, Davis JG, Shean K, Farley $J$. Serious treatment related adverse drug reactions amongst antiretroviral naïve MDR-TB patients. PLoS One. 2013;8(4):e58817. https://doi.org/10.1371/journal.pone.0058817

31. Putri FA, Burhan E, Nawas A, Soepandi PZ, Sutoyo DK, Agustin H, et al. Body mass index predictive of sputum culture conversion among MDR-TB patients in Indonesia. Int J Tuberc Lung Dis. 2014;18(5):56470. https://doi.org/10.5588/ijtld.13.0602 